\title{
MALLAMPATI TEST AS A PREDICTOR OF LARYNGOSCOPIC VIEW
}

\author{
Milan Adamus*a, Sarka Fritscherova ${ }^{\mathrm{a}}$, Lumir Hrabalek ${ }^{\mathrm{b}}$, Tomas Gabrhelik ${ }^{\mathrm{a}}$, \\ Jana Zapletalova ${ }^{\mathrm{c}}$, Vladimir Janout ${ }^{\mathrm{d}}$
}

\author{
a Department of Anesthesiology and Resuscitation, University Hospital Olomouc and Faculty of Medicine and Dentistry, \\ Palacky University Olomouc, Czech Republic \\ ${ }^{b}$ Department of Neurosurgery, University Hospital and Faculty of Medicine and Dentistry, Palacky University \\ c Department of Medical Biophysics, Faculty of Medicine and Dentistry, Palacky University \\ d Department of Preventive Medicine, Faculty of Medicine and Dentistry, Palacky University \\ E-mail:milan.adamus@seznam.cz
}

Received: July 23, 2010; Accepted: October 13, 2010

Key words: Mallampati test/Cormack-Lehane grade/Laryngoscopy/Difficult intubation/Prediction

Aim. To determine the accuracy of the modified Mallampati test for predicting difficult tracheal intubation.

Design. A cross-sectional, clinical, observational, non-blinded study. A quality analysis of anesthetic care.

Setting. Operating theatres and department of anesthesiology in a university hospital.

Material and methods. Following the local ethics committee approval and patients' informed consent to anesthesia, all adult patients (> $18 \mathrm{yrs}$ ) presenting for any type of non-emergency surgical procedures under general anesthesia requiring endotracheal intubation were enrolled. Prior to anesthesia, Samsoon and Young's modification of the Mallampati test (modified Mallampati test) was performed. Following induction, the anesthesiologist described the laryngoscopic view using the Cormack-Lehane scale. Classes 3 or 4 of the modified Mallampati test were considered a predictor of difficult intubation. Grades 3 or 4 of the Cormack-Lehane classification of the laryngoscopic view were defined as impaired glottic exposure. The sensitivity, specificity, positive and negative predictive value, relative risk, likelihood ratio and accuracy of the modified Mallampati test were calculated on $2 \times 2$ contingency tables.

Results. Of the total 1,518 patients enrolled, 48 had difficult intubation (3.2\%). We failed to detect as many as $35.4 \%$ patients in whom glottis exposure during direct laryngoscopy was inadequate (sensitivity 0.646). Compared to the original article by Mallampati, we found lower specificity (0.824 vs. 0.995$)$, lower positive predictive value (0.107 vs. 0.933), higher negative predictive value (0.986 vs. 0.928), lower likelihood ratio (3.68 vs. 91.0) and accuracy (0.819 vs. 0.929).

Conclusion. When used as a single examination, the modified Mallampati test is of limited value in predicting difficult intubation.

\section{INTRODUCTION}

Difficult/failed intubation is a significant source of morbidity and mortality in surgical patients. Despite the availability of supraglottic devices for securing the airway (LMA, laryngeal mask airway) or equipment based on sophisticated technologies (fiberoptic bronchoscopy), the inability to maintain efficient ventilation is still a lifethreatening situation for the patient. If, after induction of general anesthesia, adequate oxygenation is impossible ("cannot intubate, cannot ventilate"), urgent cricothyrotomy may be life-saving.

Unanticipated difficult intubation can be a surprising and risky operation for the anesthesiologist. Although all anesthetic departments have guidelines for the management of difficult intubation ("failed-intubation drill"), during any induction of anesthesia, the anesthesiologist must be prepared to handle difficult airways.

If we are able to predict potential difficult intubation during the pre-anesthetic visit, an alternative approach to the airway may be used from the very beginning of anesthesia, so that the risk of hypoxemia associated with difficult intubation is reduced.

The Mallampati test ${ }^{1,2}$ is one of the most frequently used clinical scoring systems to predict difficult intubation. The ease of tracheal intubation is determined by many factors, with the laryngoscopic view being the most important one. This study assessed the value of the Mallampati test as a single parameter for predicting impaired glottic exposure during direct laryngoscopy.

\section{MATERIALS AND METHODS}

Local ethics committee approval was obtained. This cross-sectional observational study was considered an assessment of the quality of care and did not alter the routine of our department. The patients gave general informed consent to the anesthesia.

During a two-month period, all adult patients (older than 18 years) presenting for any type of non-emergency surgical procedures under general anesthesia requiring 
endotracheal intubation were enrolled. Excluded were patients undergoing emergency surgery and those indicated for rapid sequence induction or scheduled for fiberoptic tracheal intubation (unstable cervical spine, previous impossible intubation, tumors of the oropharyngeal region). Patients with airway control other than endotracheal intubation were not studied either.

On arrival at the operating theatre, the airway was assessed according to the visible pharyngeal structures. We used the method described by Mallampati et al. ${ }^{2}$ and modified by Samsoon and Young ${ }^{3}$ (modified Mallampati test, MMT). On examination, the patients were seated with the head in the neutral position and the tongue maximally protruded from the mouth without phonation. We specified Mallampati Classes 1 and 2 as anticipated easy airways, and Classes 3 and 4 as predicted difficult airways $^{4}$ - see Table 1.

Following induction of general anesthesia and muscle relaxation, direct laryngoscopy was performed. The laryngoscopic view under optimal conditions ("morningair sniffing position") was described using the Cormack and Lehane ${ }^{5}$ grading system - see Table 2. For the purposes of this study, unanticipated difficult intubation was defined as difficult laryngoscopy with inadequate glottis exposure (corresponding to Grades 3 or 4 in the Cormack and Lehane laryngoscopic view).

Sex, age, weight and height were noted from the anesthetic records as well as the experience (length of practice) of the physician responsible for the anesthesia.

The relationship between the MMT (= predictor) and laryngoscopic view (= predicted parameter) was calculated with Fisher's exact test on a $2 \times 2$ contingency table. We determined the relative sensitivity, specificity, positive (PPV) and negative (NPV) predictive values, relative risk, likelihood ratio for a positive result, and accuracy. The calculations were done with the InStat v. 3.10 statistical software package (Fisher's exact test, Mann-Whitney U test, GraphPad Software, USA, www.graphpad.com) and a $P$-value less than 0.05 was considered significant. Data from the original article by Mallampati et al. ${ }^{2}$ were also analyzed using the same statistical tests and compared with our results.

Statistical terminology used in the study

1. True positive $(T P)=$ difficult intubation that had been predicted to be difficult.

2. False positive $(F P)=$ easy intubation that had been predicted to be difficult.

3. True negative $(T N)=$ easy intubation that had been predicted to be easy.

4. False negative $(F N)=$ difficult intubation that had been predicted to be easy.

5. Sensitivity = percentage of correctly predicted difficult intubations as a proportion of all intubations that were truly difficult $[=T P /(T P+F N)]$.

6. Specificity $=$ percentage of correctly predicted easy intubations as a proportion of all intubations that were truly easy $[=T N /(T N+F P)]$.

7. Positive predictive value $(P P V)=$ percentage of correctly predicted difficult intubations as a proportion of all predicted difficult intubations $[=T P /(T P+F P)]$.

8. Negative predictive value $(N P V)=$ percentage of correctly predicted easy intubations as a proportion of all predicted easy intubations $[=T N /(T N+F N)]$.

9. Relative risk $(R R)=$ ratio of the probability of difficult intubation occurring in patients with modified Mallampati test Class 3 or 4 vs. those with modified Mallampati test Class 1 or 2.

Table 1. The Mallampati test ${ }^{2}$ as modified by Samsoon and Young ${ }^{3}$ (modified Mallampati test). The visibility of the pharyngeal structures is assessed in the awake sitting patient with maximum mouth opening and tongue protrusion.

\begin{tabular}{|l|l|c|}
\hline & \multicolumn{1}{|c|}{ Visible structures } & Predicted intubation \\
\hline Class 1 & soft palate, fauces, uvula and pillars & easy \\
\hline Class 2 & soft palate, fauces and base of uvula & difficult \\
\hline Class 3 & soft palate & hard palate \\
\hline Class 4 & h
\end{tabular}

Table 2. The Cormack and Lehane grading ${ }^{5}$ of laryngoscopic view during direct laryngoscopy.

\begin{tabular}{|l|l|c|}
\hline & Laryngeal view during direct laryngoscopy & Glottis exposure \\
\hline Grade 1 & full view of glottis (vocal cords visible) & \multirow{2}{*}{ adequate } \\
\hline Grade 2 & partial view of vocal cords (anterior commissure not visible) & \multirow{2}{*}{ inadequate } \\
\hline Grade 3 & view of epiglottis, no vocal cords visible & \\
\hline Grade 4 & view of soft palate, no epiglottis visible & \\
\hline
\end{tabular}


10. Likelihood ratio for a positive result $=$ indicates how many times the difficult intubation is more likely if it is predicted with the modified Mallampati test.

11. Accuracy = percentage of correct results (both true positives and true negatives) as a proportion of all intubations $[=(T P+T N) /(T P+T N+F P+F N)]$.

\section{RESULTS}

A total of 1,518 patients were enrolled in the study. 755 (49.7\%) were males and 763 (50.3\%) were females. The demographic data (mean age, weight, height, and BMI [body mass index]) are summarized in Table 3.

Correlation between the modified Mallampati test and glottis exposure (the Cormack and Lehane grading of laryngoscopic view) is shown in Table 4, which also compares our results with those from the original article by Mallampati et al. ${ }^{2}$.
There was no correlation between the length of the anesthesiologist's practice and his/her assignments of patients to either modified Mallampati classes or laryngoscopic view grades.

\section{DISCUSSION}

The Mallampati test ${ }^{1,2}$ is a worldwide used scoring system for predicting difficult intubation. It was introduced in 1985 and has been cited in numerous publications since then. However, the accuracy of the Mallampati test has been questioned a number of times and there is controversy about its value $\mathrm{e}^{4,6-8}$. On the other hand it still remains a clinical assessment method that many anesthesiologists rely on.

In an extensive systematic review ${ }^{4}$ on 34,513 patients in 42 studies, Lee et al. found poor to good accuracy of the Mallampati test. The weak point of this meta-analysis

Table 3. Patient demographics.

\begin{tabular}{|l|c|c|c|}
\hline & Males $(\mathrm{n}=755)$ & Females $(\mathrm{n}=763)$ & $P$-value \\
\hline Age $(\mathrm{yr})$ & $51.2(17.6)$ & $51.1(16.7)$ & 0.8078 \\
\hline Weight $(\mathrm{kg})$ & $85.8(15.1)$ & $74.3(16.2)$ & $<0.0001$ \\
\hline Height $(\mathrm{cm})$ & $177.7(7.5)$ & $165.3(6.3)$ & $<0.0001$ \\
\hline BMI $\left(\mathrm{kg} \mathrm{m}^{-2}\right)$ & $27.13(4.37)$ & $27.18(5.75)$ & 0.3916 \\
\hline
\end{tabular}

Data are mean and standard deviation, Mann-Whitney U test was used for calculations.

Table 4. Correlation between the modified Mallampati test and laryngoscopic view (glottis exposure) graded with the Cormack and Lehane score.

\begin{tabular}{|l|c|c|c|}
\hline & $\begin{array}{c}\text { Our results (modified } \\
\text { Mallampati test) }\end{array}$ & Mallampati et al ${ }^{2,4}$ & $P$-value \\
\hline $\mathrm{n}$ & 1,518 & $14(6.7 \%)$ & 0.0005 \\
\hline True positive (n) & $31(2.0 \%)$ & $1(0.5 \%)$ & $<0.0001$ \\
\hline False positive (n) & $258(17.0 \%)$ & $181(86.2 \%)$ & 0.0318 \\
\hline True negative (n) & $1,212(79.8 \%)$ & $14(6.7 \%)$ & $<0.0001$ \\
\hline False negative (n) & $17(1.1 \%)$ & $0.500(0.306$ to 0.694$)$ & $<.235$ \\
\hline Sensitivity & $0.646(0.494$ to 0.778$)$ & $0.995(0.970$ to 1.000$)$ & $<0.0001$ \\
\hline Specificity & $0.824(0.805$ to 0.843$)$ & $0.933(0.681$ to 0.998$)$ & $<0.0001$ \\
\hline Positive predictive value & $0.107(0.074$ to 0.149$)$ & $0.928(0.883$ to 0.960$)$ & $>0.05$ \\
\hline Negative predictive value & $0.986(0.978$ to 0.992$)$ & $13.00(7.71$ to 21.92$)$ & $<0.05$ \\
\hline Relative risk & $7.76(4.35$ to 13.82$)$ & $91.0(12.45$ to 665.31$)$ & $<0.0001$ \\
\hline Likelihood ratio+ & $3.68(2.90$ to 4.66$)$ & $0.929(0.885$ to 0.959$)$ & \\
\hline Accuracy & $0.819(0.799$ to 0.837$)$ & & \\
\hline
\end{tabular}

The $95 \%$ confidence intervals are in parentheses. Data calculated from the original article by Mallampati ${ }^{2}$ are also presented. The $P$-value demonstrates the differences between our and Mallampati's results (Fisher's exact test used). 
is the fact that sometimes the complete data from the original articles are not available or they may be of different quality due to dissimilar criteria used.

The original Mallampati test ${ }^{2}$ used three classes (Class 1 - faucial pillars, soft palate and uvula could be visualized, Class 2 - faucial pillars and soft palate could be visualized but the uvula was masked by the base of the tongue, Class 3 - only soft palate could be visualized). The modification of Samsoon and Young ${ }^{3}$ (MMT) describes four classes (Table 1) and today, this scoring system is usually referred to when talking about the Mallampati test.

The laryngoscopic view grading may be differently described in the literature, too. Mallampati originally used a four-grade scale ${ }^{1,2}$ (Grade 1 - glottis could be fully exposed, Grade 2 - glottis could be partly exposed [anterior commissure not visualized], Grade 3 - glottis could not be exposed [corniculate cartilages only could be visualized], Grade 4 - glottis including corniculate cartilages could not be exposed). Grades 1 and 2 were considered "adequate exposure" and Grades 3 and 4 "inadequate exposure." For our study, we used the most commonly applied scoring system by Cormack and Lehane ${ }^{5}$. This also consists of four grades, however, Grades 3 and 4 are somewhat different (Table 2) from the original scale by Mallampati et al. ${ }^{2}$.

Although the original article by Mallampati et al. ${ }^{2}$ reported neither positive predictive value nor sensitivity or specificity, it contained raw data. These were used for calculating the appropriate statistical values to be compared with our study (Table 4). We could not confirm the optimistic results of Mallampati et al. ${ }^{2}$. The two studies must be compared with caution despite the fact that they deal with the same problem (prediction of laryngoscopic view). In our study, slightly different criteria were used for describing both the quality of the laryngeal view and the visibility of the oropharyngeal structures. This can be an additional source of different results.

The modest degree of accuracy is not the only limitation of the modified Mallampati test. There are a large number of other factors that may be based on improper clinical assessment. The inter-observer reliability of the MMT can be poor (kappa as low as 0.31$)^{9}$. The visibility of the oropharyngeal structures also depends on the patient's position during examination. The classical Mallampati test is done in the sitting patient, with his/her head in the neutral position. However, when performed in the supine position, the test may have a higher positive predictive value and is associated with more true positives than assessment in the sitting position ${ }^{10-12}$. The specificity and positive predictive value of the MMT can be improved when performed in the sitting patient with extension of the craniocervical junction ${ }^{13}$. MMT is also influenced by the patient's ethnic group: tracheal intubation may be more difficult in Asian than Caucasian patients ${ }^{14}$. The incidence of modified Mallampati Classes 3 and 4 also increases during labor compared with the pre-labor pe$\operatorname{riod}^{15}$, and these changes are not fully reversed within 48 hours after delivery ${ }^{16}$.

Our study has several limitations that are primarily due to logistic conditions. All the data were collected dur- ing a two-month anesthetic care provided in busy operating theatres. The examinations were not blinded; the same anesthesiologist assessed both the modified Mallampati test and laryngoscopic view. Forty-five anesthesiologists with different expertise were involved in assessing the patients and this may be a potential source of bias.

On the other hand, this also reflects the heterogeneity of routine clinical practice, which may paradoxically be considered an advantage of the study ${ }^{13}$. However, we could not demonstrate a correlation between the length of the anesthesiologist's practice and his/her assignments of patients to either modified Mallampati classes or laryngoscopic view grades.

We used impaired laryngoscopic view as a sign of difficult intubation. The definition of difficult intubation is usually based on the presence or absence of several features. These may include total preclusion of intubation, number of intubation attempts, easy advancement of the endotracheal tube into the trachea and duration of intubation. However, the quality of the laryngoscopic view during direct laryngoscopy is considered the most important factor.

We studied a larger group (1,518 patients) than Mallampati et al. ${ }^{2}$ (210 patients). In their study, Mallampati et al. reported a higher proportion of patients with inadequate glottis exposure (28 cases out of 210 patients, $13.3 \%$ ) compared to our results ( 48 cases out of 1,518 patients, $3.2 \%$; $<0.0001$ ).

Using the MMT, we failed to detect as many as $35.4 \%$ patients in whom the glottis exposure during direct laryngoscopy was found inadequate $(64.6 \%$ sensitivity). The specificity was higher in the original article than in ours (99.5\% vs. 82.4\%; p < 0.0001$)$.

Sensitivity and specificity are dependent parameters with negative correlation. When the sensitivity increases, the specificity usually decreases and vice versa. The preanesthetic examination of the airways should be primarily aimed at detecting as many patients with difficult airways as possible. This is necessary for minimizing the risk of unanticipated difficult/failed intubation ${ }^{17}$. From this point of view, the sensitivity of a test may be a more valuable parameter for predicting difficult intubation than its specificity.

From the data in the original article by Mallampati ${ }^{2}$, very high positive and negative predictive values could be calculated. While the NPV in our study was even higher than in the original article (98.6\% vs. 92.8\%; $\mathrm{p}<0.0001)$, the PPV was significantly lower $(10.7 \%$ vs. 93.3\%; $<<0.0001)$. This demonstrates that compared to Mallampati's article, there was a lower percentage of correctly predicted difficult intubations as a proportion of all predicted difficult intubations (PPV), and a higher percentage of correctly predicted easy intubations as a proportion of all predicted easy intubations (NPV).

The test for predicting difficult intubation should have high accuracy, sensitivity and positive predictive value to identify all patients in whom intubation will be difficult ${ }^{17}$. Apparently, no clinical test, the Mallampati scoring system being no exception, can meet these criteria when used alone. Effective and reliable prediction requires a com- 
bination of several parameters (e.g. age, height, weight, BMI, MMT, head and neck movement, dentition status, upper lip bite test ${ }^{18-20}$, interincisor gap ${ }^{17,21,22}$, and thyromental distance $\left.{ }^{17,22,23}\right)$. Usually, the more parameters are used, the higher is the accuracy of prediction. On the other hand, it is impractical to perform a large number of time consuming tests before general anaesthesia. The ongoing research aimed to identify the factors and their combinations that are relevant for prediction. So far, a number of models for predicting unanticipated difficult intubation have been described ${ }^{17,21,22}$, but none have a $100 \%$ accuracy. It follows therefore that the anesthesiologist must always be prepared to manage difficult airway when such a problem arises.

\section{CONCLUSION}

The modified Mallampati test is a simple clinical assessment to predict inadequate exposure of the glottis and consequently difficult tracheal intubation. When used alone, it is of limited value and cannot be relied on.

\section{ACKNOWLEDGEMENTS}

The authors gratefully acknowledge financial support from the Czech Ministry of Health Internal Grant Agency project No. NS 9618-4/2008.

The authors express their sincere thanks to anesthesiologists and nurses from the Department of Anesthesiology and Resuscitation, University Hospital Olomouc, Czech Republic, for their help with data collection.

\section{REFERENCES}

1. Mallampati SR. Clinical sign to predict difficult tracheal intubation (hypothesis). Can Anaesth Soc J 1983;30:316-7.

2. Mallampati SR, Gatt SP, Gugino LD, Desai SP, Waraksa B, Freiberger D, Liu PL. A clinical sign to predict difficult tracheal intubation: a prospective study. Can Anaesth Soc J 1985;32:429-34.

3. Samsoon GL, Young JR. Difficult tracheal intubation: a retrospective study. Anaesthesia 1987;42:487-90.

4. Lee A, Fan LT, Gin T, Karmakar MK, Ngan Kee WD. A systematic review (meta-analysis) of the accuracy of the Mallampati tests to predict the difficult airway. Anesth Analg 2006;102:1867-78.

5. Cormack RS, Lehane J. Difficult tracheal intubation in obstetrics. Anaesthesia 1984;39:1105-11.
6. Cattano D, Panicucci E, Paolicchi A, Forfori F, Giunta F, Hagberg C. Risk factors assessment of the difficult airway: an Italian survey of 1956 patients. Anesth Analg 2004;99:1774-9.

7. Shiga T, Wajima Z, Inoue T, Sakamoto A. Predicting Difficult Intubation in Apparently Normal Patients: A Meta-analysis of Bedside Screening Test Performance. Anesthesiology 2005;103:429-37.

8. Karkouti K, Rose DK, Wigglesworth D, Cohen MM. Predicting difficult intubation: a multivariable analysis. Can J Anaesth 2000; 47:730-9.

9. Karkouti K, Rose DK, Ferris LE, Wigglesworth DF, Meisami-Fard T, Lee H. Inter-observer reliability of ten tests used for predicting difficult tracheal intubation. Can J Anaesth 1996;43:554-9.

10. Bindra A, Prabhakar H, Singh GP, Ali Z, Singhal V. Is the modified Mallampati test performed in supine position a reliable predictor of difficult tracheal intubation? J Anesth 2010;24:482-5.

11. Tham EJ, Gildersleve CD, Sanders LD, Mapleson WW, Vaughan RS. Effects of posture, phonation and observer on Mallampati classification. Br J Anaesth 1992;68:32-8.

12. Singhal V, Sharma M, Prabhakar H, Ali Z, Singh GP. Effect of posture on mouth opening and modified Mallampati classification for airway assessment. J Anesth 2009;23:463-5.

13. Mashour GA, Kheterpal S, Vanaharam V, Shanks A, Wang LY, Sandberg WS, Tremper KK. The extended Mallampati score and a diagnosis of diabetes mellitus are predictors of difficult laryngoscopy in the morbidly obese. Anesth Analg 2008;107:1919-23.

14. Wong SH, Hung CT. Prevalence and prediction of difficult intubation in Chinese women. Anaesth Intensive Care 1999;27:49-52.

15. Pilkington S, Carli F, Dakin MJ, Romney M, De Witt KA, Doré CJ, Cormack RS. Increase in Mallampati score during pregnancy. Br J Anaesth 1995;74:638-42.

16. Boutonnet M, Faitot V, Katz A, Salomon L, Keita H. Mallampati class changes during pregnancy, labour, and after delivery: can these be predicted? Br J Anaesth 2010;104:67-70.

17. Naguib M, Scamman FL, O'Sullivan C, Aker J, Ross AF, Kosmach $\mathrm{S}$, Ensor JE. Predictive performance of three multivariate difficult tracheal intubation models: a double-blind, case-controlled study. Anesth Analg 2006;102:818-24.

18. Tremblay MH, Williams S, Robitaille A, Drolet P. Poor visualization during direct laryngoscopy and high upper lip bite test score are predictors of difficult intubation with the GlideScope videolaryngoscope. Anesth Analg 2008;106:1495-500.

19. Myneni N, O'Leary AM, Sandison M, Roberts K. Evaluation of the upper lip bite test in predicting difficult laryngoscopy. J Clin Anesth 2010;22:174-8.

20. Eberhart LH, Arndt C, Cierpka T, Schwanekamp J, Wulf H, Putzke C. The reliability and validity of the upper lip bite test compared with the Mallampati classification to predict difficult laryngoscopy: an external prospective evaluation. Anesth Analg 2005; 101:284-9.

21. Wilson ME, Spiegelhalter D, Robertson JA, Lesser P. Predicting difficult intubation. Br J Anaesth 1988;61:211-6.

22. Arné J, Descoins P, Fusciardi J, et al. Preoperative assessment for difficult intubation in general and ENT surgery: predictive value of a clinical multivariate risk index. Br J Anaesth 1998;80:140-6.

23. Butler PJ, Dhara SS. Prediction of difficult laryngoscopy: an assessment of the thyromental distance and Mallampati predictive tests. Anaesth Intensive Care 1992;20:139-42. 
